\title{
Advancements in Radiographic Evaluation Through the Migration into NDE 4.0
}

\author{
Hossain Saboonchi ${ }^{1}$ (I) . Donald Blanchette ${ }^{2} \cdot$ Kimberley Hayes $^{3}$
}

Received: 31 July 2020 / Accepted: 5 January 2021 / Published online: 23 January 2021

(c) The Author(s), under exclusive licence to Springer Science+Business Media, LLC part of Springer Nature 2021

\begin{abstract}
The challenges presented by the global pandemic and slump in oil prices have imposed costly avoidance measures and delayed project timeliness, but it also has created the opportunity for innovation conditions in industrial non-destructive testing. The evolutional path leveraged by Industry 4.0, present sustainable offerings of robotic platforms, digital solutions and connected devices commonly known as the Internet of Things (IoT) that may assist in recapturing some of the current losses. The landscape is broad with staggered adoptions. An overview of the Industrial Revolutions, related developments in NDE 4.0 and specific focus on North American radiography in the petroleum industry is highlighted. Additionally, focusing on the importance of shared transparency and burden throughout the value-chain to ensure efficacy throughout the migration, and the human contributor for collaborative transition and skills required for the future. The evolutional path of Industrial revolutions leads to Industry 4.0 which presents opportunities with Artificial Intelligence, and connected devices commonly known as the Internet of Things (IoT). This article focuses on three major issues for consideration in the development of a strategic plan to capitalize on the advancements of digital radiography in the petroleum sector. The components are the technological NDE 4.0 transformation, the need for a new and realistic perspective of digital radiography, and the challenges of developing a workforce to adapt to the transition of NDE 4.0.
\end{abstract}

Keywords Nondestructive evaluation (NDE) $\cdot$ NDE 4.0 $\cdot$ Radiographic testing $\cdot$ Human factor $\cdot$ Technology integration

\section{Industry 4.0: Evolution of Technology}

\subsection{Background}

Revolution in science, society and government can have a birthdate where one can clearly see the time before and after, like the development of penicillin, or Arab spring; however, industrial revolutions evolve over long periods and are often marked by the decades that they blossomed and grew into wider adoption, at least in the industrialized societies.

The design and use of first steam engines can be used as an indicator for 1st industrial revolution, i.e., Industry 1.0,

Hossain Saboonchi

hossain.saboonchi@mistrasgoup.com

1 Product \& System Div, MISTRAS Group Inc., Princeton Junction, NJ, USA

2 Training Department, MISTRAS Group Inc., Princeton Junction, NJ, USA

3 VMI, Houston, TX, USA in the late eighteenth century. By the late nineteenth century, adoption of mass production in factories and the introduction of electrically powered assembly lines are often considered the turning points in the price of goods reduction and are considered the 2nd industrial revolution or Industry 2.0. The birth of personal computers, miniaturized electronics, and the use of automated robotics in industry form Industry 3.0 in the 1970s $[1,2]$.

\subsection{Industry 4.0}

In recent years, many different buzz words promise the 4th revolution in industry, including; Artificial Intelligence (AI), advanced robotics, automation, and virtual reality. Each of these tools was enabled by powerful computers with larger and cheaper data storage capacities, taking the form of remote supercomputers or cloud computers. These technologies are revolutionizing industries including retail, transportation, banking, medicine, and manufacturing. While the changes were significant in some cases, neither one was significant enough to become the anchor point of Industry 
4.0. Following these initial deployments, some revolutionary technologies like blockchain and crypto-currencies, additive manufacturing, 3D printing, and finally production of low cost connected systems often in the form of a data collection system known as the Internet of Things (IoT) makes the prospect of another revolution greater [3].

Those trying to define what Industry 4.0 is are mainly referring to a connected digital world that is correlated with the physical world $[1,2,4-6]$. This correlation, often described as digital model, which monitors and controls its physical twin and it is often referenced as "Digital Twin". The digital twin correlation can be manifested in smart homes, autonomous vehicles, and industry. It can be an autonomous distribution warehouse or a modern refinery with 24/7 continuous monitoring and control of the process and assets. In most of these cases, the digital twin is made up of data gathering and a display dashboard, as well as, sophisticated computer models to comprehend the data, analyze it, and make decisions based on predefined requirements and rules to optimize the performance of the asset [7]. Figure 1 visualizes the relationship between a physical asset and its "Digital Twin" and how they interact.

\subsection{NDE 4.0}

NDE 4.0 proposes connectivity on every level from measurement and testing of the asset, to storage and transfer of data, to data analysis and decision making, to reporting and consecutive actions by the asset owner or its proxies and vendors. Traditional communication is challenged by modern digital pipelines of data. The infrastructure of this communication is evolving very rapidly, and as it does, the cost of modern platforms is continually decreasing. This reduction in expense will force industries to adapt or become outdated, and in extreme cases extinct. In this section, we will look at the effect of modern connectivity on the different aspects of Non-Destructive Examination (NDE) processes.

NDE imaging, using modern equipment like digital radiography, captures images in a digital form that is ready to

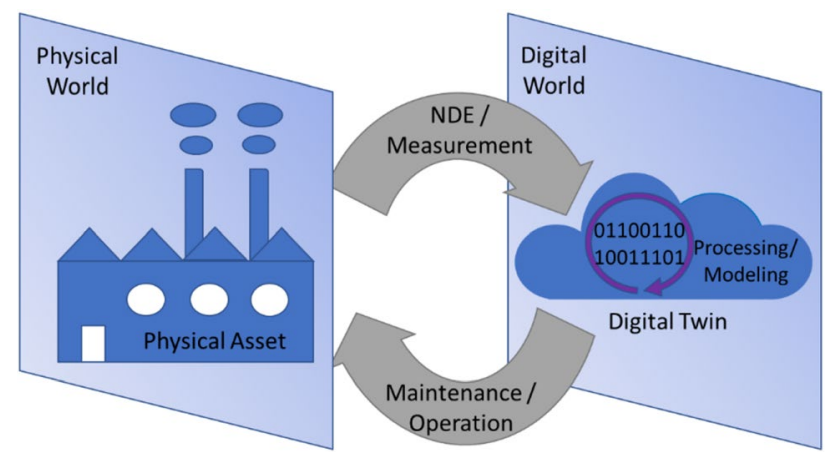

Fig. 1 Schematic diagram of the Digital Twin for NDE 4.0 be stored on computers. This enables real-time analysis and connectivity of the information from the test site to the endusers. An inspector evaluates the quality of the asset; asset owners can track the history of the component and commission needed repairs; and auditors can audit the NDE vendor and asset owners to see if they meet the regulations and standards.

\section{Industrial Technology Enabling Migration}

\subsection{Connectivity}

There are various NDE connectivity technologies, which permit communication using conventional methods like Wireless Fidelity (WiFi) and cellular to exotic methods; such as, satellite communication. Connectivity is an important element in the evolution of the industrial world. There are many secure Low Power Wide Area Network (LPWAN) in the market claiming optimal connectivity for the IIoTs, including the most well-known commercial protocols LoRa, LoRaWAN, SigFox, WirelessHART, NB-IOT, CAT M1 [8, 9].

\subsection{Cloud Computers}

Next, data needs to be stored and be available to multiple end-users. Common communication tools like emails are not suitable for transfer of large data sets or sensitive data from inspection of assets. The solution to this "big data" issue is access to powerful servers connected to the internet with user access privileges, encryption, and with an abundant supply of resources like computation, storage, and bandwidth, commonly called "cloud computers". The commercially available clouds are expanding their capabilities and reducing their price and risks, making them viable option. Some of the most famous commercial clouds are Amazon's AWS (Amazon Web Services), Google's GCP (Google Cloud Platform), Microsoft's Azure, and IBM's Watson or Maximo. These platforms have varying degrees of services including file-sharing, SharePoint, web hosting, databases, AI tools, computational resources, security and privacy protection tools, and most importantly different data storage mediums with features like archival and retrieval speed, back up periods, and costs.

\subsection{Data Formats}

There is no platform or technology which has dominated the NDE world and specifically for radiography techniques. However, the pioneers in image analysis and Industry 4.0 have been active in the medical field and lay precedence for a potential path. Many companies and universities have 
been working on Assisted Diagnostics and screening tools for physicians in the medical world. A widely accepted standard for imaging in the medical field is called DICOM (Digital Imaging and Communications in Medicine) [10]. These techniques and standards have been suggested for NDE applications. A convergence on one format has centered around DICONDE (Digital Imaging and Communication in Nondestructive Evaluation) to support a unified data exchange by The American Society for Testing and Materials (ASNT) through E2339-15 Standard Practice for DICONDE [11]. However, there has been no concrete movement in that direction by technical associations like ASME (The American Society of Mechanical Engineers), or API (The American Petroleum Institute), or ANSI (American National Standards Institute).

\subsection{Artifactual Intelligence}

Artifactual Intelligence (AI) and its subfields like Machine Learning (ML) have upended many industries traditional approaches. The Assisted Driving and in its ultimate form Autonyms Driving in automotive industry [12], Auto Pilot for Aviation Industry [13], and the Assisted Diagnostics for MRI, CT, and X-ray images in medical field [14-18] are just some examples of how this technology is revolutionizing industries and assisting professionals by increasing their accuracy and reducing the risks associated with human errors. In all these examples human life is at stake and any error in algorithms could have catastrophic consequences. Additionally, many regulatory frameworks have to be updated to accept this technology and define the liabilities and acceptance criteria.

Adoption of AI based tools for NDE and Structural Health Monitoring (SHM) has already initiated by many works in academic world [19-23] and some industry initiatives [7]. However, wide transition of these tools to NDE industries is slower than desired. There are still many challenges to be overcome including Verification and Validation of the performance of these tools and techniques for many different NDE methods and industries, integration of these tools into the governing codes and standards and into NDE equipment and infrastructures.

\subsection{Codes and Standards}

The adoption of any change by technical associations has many folds, including providing a reliable and clear technical roadmap of what is acceptable, and how data and reports should be prepared and archived. Also, there is the legal aspect in terms of liability of different parties involved, training required for the technicians, level I, II, and III inspectors, as well as, the auditors' knowledge which are set by technical associations. Many technical associations including but not limited to ISO, ANSI, ASNT, ASME, and API are involved and each one will define some aspect of this transformation.

While some technical associations have already developed codes and standards for digital radiography, there is a lack of awareness in the NDE community on what NDE 4.0 and connected nature of the new era entails. One can argue that this an issue beyond NDE and should be addressed by other technical associations like IEEE (The Institute of Electrical and Electronics Engineers) and IEC (The International Electrotechnical Commission) as they are the true experts of that aspect of data and its handling, just like how OSHA (Occupational Safety and Health Administration) covers the safety of personnel involved in NDE community. The counterargument has been that there is no reference to any of the standards developed by associations like IEEE or IEC and there are gaps in terms of standard data structures for different NDE techniques.

For example, in the case of radiography as an NDE technique, the NDE radiography community must adopt any of the available standards or develop one in collaboration with the experts of those fields if none of the existing ones serve the NDE community's needs. Other gaps may include standards for privacy and security of digital data, compression model/file format to handle the image files, and any naming and scaling conventions that could be utilized for data archival of the digital images. In lieu of accepted guidelines by the technical associations, it is up to the hardware manufacturers, service providers, and asset owners to choose the framework of adoption. This makes switching from one hardware to the next or this vendor the next a costly transformation, which would prevent the asset owners from adopting the new technology.

\section{Market Migration in Industrial Radiography}

In this paper we will focus on the Radiography in the oil and gas industry, however we acknowledge that similar comparisons and study could be done for different industries and their specific application and journey into the NDE 4.0. Market projections present a level of optimism in availability of NDE technology, but there is a gap in ideology and path to adoption. Businesswire 2019 published, "the global IoT in the oil and gas market is expected to reach $\$ 43.48$ billion by 2024, rising at a CAGR of $21.86 \%$ from 2019 to 2024" [24]. While Gartner also revealed that " $53 \%$ of oil and gas companies have failed to implement IoT technology and are not planning to do so" [25]. It invites the question of "Why?" A potential reason is many of the North American refineries are between 50 and 120 years old, and Gartner also states a key cause for the delayed adoption is "Evolving strategies 
and intensifying digital ambitions have rendered the oil and gas IT operating models unfit for purpose" [26].

To bridge the gap and capitalize on NDE 4.0, there must be a collaborative charter that is pushed throughout the value-chain for seamless transition to ensue stepwise iterations for the collective good. A clear messaging from the asset owners of their vision supporting the ideal condition in conjunction with value-stream, including the onsite inspection company, the equipment manufacturer to optimize system performance is needed to standardize potential solutions. The collaborative approach invokes an industry-wide adoption and commitment to change while sharing through honest transparency and burden in cost of change management throughout the value-chain with primary importance on dataflow and fidelity. The value-channel may be different if addressing Mechanical Integrity issues on existing legacy facilities or planning, construction of expansion/ new projects.

With legacy sites, the battle of current infrastructure may take incremental adaptations where collaborative dialogue with owner/operators, inspection service providers, equipment manufacturers, IT, etc. to manage the Capital Expenditures and valuation of the direct/indirect cost and efficiencies. This will cultivate a sustainable business strategy and human-factor migration throughout the evolving vision. On the other hand, Expansion/New: looking forward to the future where the potential of digital twins and digital traceability require a vision from the asset owner and collaborative transparency with the EPC (Engineering, Procurement and Construction) for design with the future ideal condition in mind. Unmanned offshore platforms needs are different from legacy but can be designed with the target in lieu of brownfield retrofitting. Codes/Standards must adapt to meet future and present needs like robot access or in situ monitoring in lieu of human entry. The development of future communication solutions must be considered with inspection hardware solution roadmap designs to present future innovation.

\subsection{Industry Challenge}

A conversation of concept, adoption and utilization still trickle down with many questions regarding the practicality of execution of "What", "Where", "When". Open dialogue and clear view to successful use cases in deployments throughout the oil, gas and petrochemical industry can escalate and support industry-wide adoption.

Some of the "what" that need definition are, cloud platform standardization and communication protocols (mentioned in earlier section) from the industry so that the value-chain (Owner/Operator, onsite inspection company and equipment manufacturers) can build a strategic development trajectory. Another key aspect will be the data linking in both directions affording a reliable data exchange to and from Risk-based Inspection (RBI) platforms where machine learning protocols can continue to evolve in accuracy and reliability.

The focus on developing "where" to prioritize specific capabilities are best directed by the asset owner with insights from the onsite inspection company's vantage to new technologies and the equipment manufacturers development roadmap. Additionally, weighing the provisions site-wide or controlled areas of the plant. In recent years, bold claims by owners requiring robotics to be used for confined space inspections, open the pathway for development and tracking for challenges that may not be suited well with current technologies. This top-down forced initiative support where industry focuses on solutions. Some opportunities are driven by regulatory bodies and governmental reporting, as seen in the pipeline industry.

The recent requirements of geospatial tracking can best be satisfied either through the inspection equipment if the needs are understood or by the service company. Working together assessing a reliable standardization can support the evolution of the industry as a whole instead of a competitive advantage which can ease in adoption and changemanagement. Evaluating it on the macro-perspective can help with solutions that may decrease project costs and timeline compressions that satisfy regulatory requirements. Another industry trend is autonomous/unmanned platforms and FPSOs (Floating, Production, Storage and Offloading) demand digitization and present controlled environments, this requires multi-disciplinary collaboration and standardization from design, fabrication to run and maintain. Progressive owners need to work with construction codes for future solutions. A 24" manway may not be necessary if the future is viewed with in situ robots in continuous inspection mode. Changes of this magnitude require drastic measure, but assurance of integrity must govern. Collectively a framework validation model must be established to open the technical landscape will clearly defining the "swim lanes" for validation. Protocols may leverage existing Performance Demonstration Qualification (PDQ) models or Probability of Detection (PoD) respective to Level of Consequence (LoC).

A third aspect of transparency must focus on the "when" aligning current capabilities of equipment, general connected technology and the strategic plans of the future. As $5 \mathrm{G}$ is still evolving in this space, the value-chain must evolve with it in synchronous progression. The simple answer of timing is now.

The collective evolution industry-wide in aligning, developing and deploying a sustainable solution must be of collaborative value. The creation of an over-arching roadmap can align hardware, software, procedures and personnel for a secure migration. 


\section{Use-Case Review}

Ideal context may be seen in conventional industrial radiographic testing as it has virtually been unchanged for the past 75 years and remains the number one method for volumetric examination while other methods like ultrasonic testing continues to evolve. While digital solutions are available, the North American market maintains an estimated share of approximately $85 \%$ film utilization. Radiography must find a path to the digital age.

It is important to embrace this dramatic transition to digital radiography through-out the value-chain (previously discussed) for adoption to be successful. The focus must shift away from lowest price per exposure or day rate bid to sustain the investments in materials, procedures, processes, and personnel. Weighing the efficiencies of the long game need to be paramount. Purchasing must align with the vision of the digital future with weighted metrics of strategic migration path as significant as safety record to motivate progressive partners.

The undervalued weight of data quality and seamless data flow, the speed in which acquisition to evaluation is attained, increased capabilities in data management, increased safety factors and global collaboration experienced through digital radiographic solutions must tilt the scales in the price discussions. The ecosystem of Digital Twin is only sustainable if the data is of high quality, seamless exchange in architecture and infrastructure and the workforce skills match the technology. The question begs, why is there such a disproportionate percentage of radiographic testing still utilizing conventional film when digital solutions exist in Computed Radiography and Digital radiography? Desire and action must converge. Expense of change may be an obstacle, but fear behind the hype of Artificial Intelligence (AI) may need demystification for adoption to ensue.

\subsection{Precedence: Banking and Medical}

Personal use-cases may help with vantage and confidence in transitioning to connected, digital data and cloud computing and demystify the reluctance as they may apply to digital radiography. Assumed confidence has been dispensed in the secure transference of sensitive information in the banking industry with shared correlation to inspection in the algorithms used that transfers funds from a photograph. The migration from paper checks at teller for deposits to current condition of scan check images is trusted, accurate and the direct transfer provides time savings and cost savings for the institution and individual.

In 2010, it was stated, mobile deposit was a thing of the future [27] and at that time broached similar concerns in NDE of fraud and the privacy of financial data, now is commonplace. Personal data privacy, accuracy in of amounts to specific locations are more aligned with industrial aspects of $\mathrm{NDE}$, if embraced. Additionally, many large banks leverage technology that use Artificial Intelligence (AI) and Machine Learning (ML) to decipher and convert handwriting into text via Optical Character Recognition (OCR) that has a long history in converting handwritten image documents to digital files of characters and other neural networks support fraud detection (Fig. 2 Script to digital Convolutional Neural Network. IEEE Gradient-based Learning Applied to Document Recognition [28]) presented in 1998 Proceedings by IEEE [28]. Connected devices and image algorithms transfers our most important personal assets both data and finances without question. Similar Convolutional Neural Networks and other Deep Learning solutions may be used to assist the technician in detecting, characterizing indication in digital radiographic examination. Similarly aligned with NDE the well-established diagnostics widely used in the medical industry for digital radiographic assessment. The benefits, security and remote review capabilities offer an understanding potential workflow and data pathway that may be used by the industrial sector. Leveraging deep learning for auto diagnostics, which is unfamiliar to many, but has established confidence it has placed in many aspects of our existence
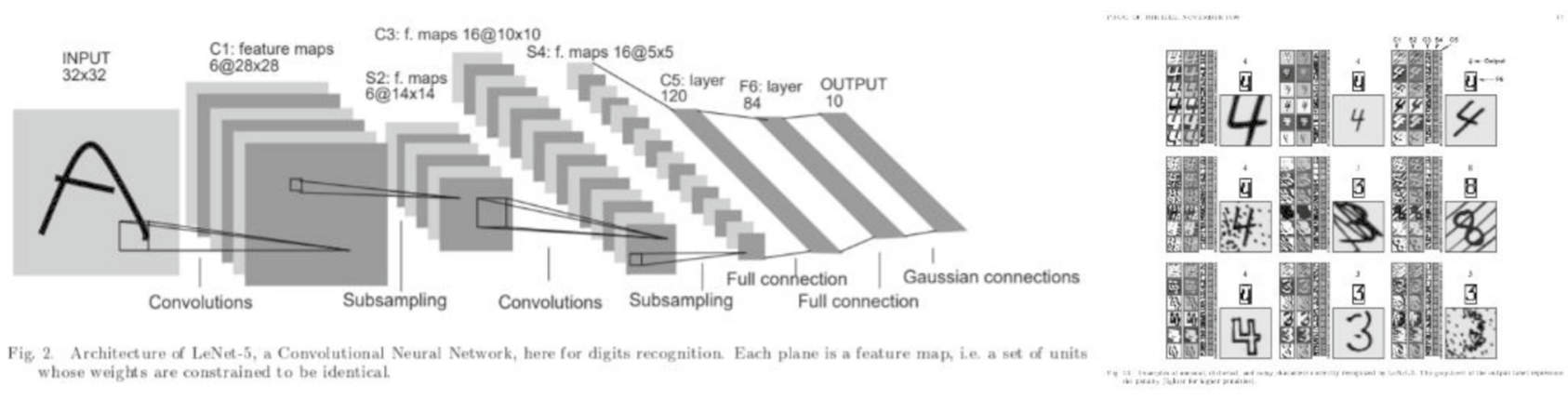

Fig. 2 Script to digital Convolutional Neural Network. IEEE gradient-based learning applied to document recognition [28] 
[14-18]. The mature aspects continue to evolve past with apprehensive utilization in NDE must be discussed and addressed.

Assessing across aspect of personal experiences and an array of markets, innovation can draw from potential solutions radiographic acquisition, evaluation, transmission, and storage. Soon it will not be a question of should, but an emphatic must adapt and adopt these technologies. Collective elevation should be for the greater good and not slated as a competitive advantage or product feature focus.

It is prudent for the value-chain to converge on a standardized validation protocol to minimize barrier to entry of the vast networks of technology, but clear vantage to the framework to assess Technical Readiness Level (TRL).

\subsection{Industrial Use-Case: Maintenance and Inspection of Legacy Sites}

One example of value-chain engagement in the migration to leverage NDE 4.0 through legacy sites in the can be recognized in the Corrosion Under Insulation (CUI) inspection. With approximately $5-10 \%$ of the overall turnaround cost allocated to people, labor, and materials (excluding cleaning and other preparation), optimizations garnered through the digital migration may exponentially offset initial investment for this transition from film. An honest evaluation in optimization to the inherent aspects of film, Computed Radiography (CR) and Digital Radiography (DR). Highlighting the benefits of migration overshadow the investment by optimizing operator repeatability, intuitive workflow management, data fidelity, digital project scheduling, no chemical management and film storage as it pertains to materials that must be affirmed to the progressive bids and contract lets. Action follows reward and the message must resonate [29, 30].

A \$2.5 trillion annual global spend by CUI has been labeled as the root cause of global industry challenges forcing shutdowns, lost production; early repair/replacement and safety and environmental consequences [31]. Radiography capability to interrogate while not requiring the cost and potential damage caused in the removal of the insulation. Current bid formatting is based around legacy use of film presenting little motivation for onsite service provider to invest in hardware and training of personnel required for digital imaging. Increased value of radiography in the film to digital migration should focus on the backend value (cost) of reliable data utilization and storage. Although not exhaustive example, the material portion of the turnaround may view the $\$ 0.85 /$ sheet of $4.5^{\prime \prime} \times 10^{\prime \prime}$ film cost in contrast to the Computed Radiographic (CR) plates can range in the $\$ 150$ per similar size plate. A holistic view must be leveraged versus low bid. Digital data affords utilized for future baselining and potential trend analysis, direct linkage to Inspection
Data Management System (IDMS) the digital data values or findings and support mitigation of human factors.

Transparent considerations should be considered with assessing the value of digital migration. Film delivers an image in an "as processed" state with limited ability to enhance for increased detection; film speed selection (D2-7) specified pre-inspection which can limit adaptability and raise another opportunity for error; single use that require chemical processing (developer, stop bath, fixer, rinse, surfactant rinse, dry) generating environmental waste; substantial time even when batch processed that delay results and film storage housing and/or disposal environmental implications of heavy metals. Where Computed Radiographic imaging plate, the cost can be amortized to present a lower cost impact. Similar price correlation as above, a $4.5^{\prime \prime} \times 10^{\prime \prime}$ averages $\sim \$ 150$ per imaging plate. With proper handling can produce upwards of 1500-2000 exposures and decrease media use to $\$ 0.10$ to $\$ 0.08$ cents/exposure with an average use of 100 exposure at $\$ 1.50$ per exposure. Much of the profitability resides in the human factors with effective IP management care from the inspectors.

In lieu of chemical processing, scanner is used excite latent information from the Imaging Plate (IP) into a digital (pixel/voxel) output that can be easily linked to IDMS and digital storage for archiving traceability and does not fade over time like film. Unlike film evaluation in "as processed" state, CR leverages the availability of image optimization techniques, like filters, resizing, compensation for lower dose in scanner settings may afford increased safety. Another solution that provides near-real-time review can be seen using Digital Detector Arrays (DDAs). Although Digital panel rigidity may limit some of the applications and may present scalability challenges initially, opportunities do exist in format linkage to digital IDMS system, immediate direct review with potential for remote and global distribution to increase reliability and decrease reshoots. Additionally, digital file storage versus hard storage are just a few of the recognized data advantages.

Both CR and DR provide environmental benefits, for example, not requiring chemical processing, management or disposal, there are associated safety benefits through source maximization in panel sensitivity allowing for lower curie strengths and alternative source selection providing for less impact on operations. Due to the non-contact to the part (unlike film, CR), can accommodate expanded temperature challenges. A very important value-proposition for conversion is the ability to evaluate with filters in post-processing. Below in Fig. 3 demonstrates the same exposure with enhancements providing opportunity for further interrogation. The left image provides enhanced view of the condition of the insulation and the far-right corrosion of piping. The data is unchanged, just enhanced to leverage viewing benefits. 


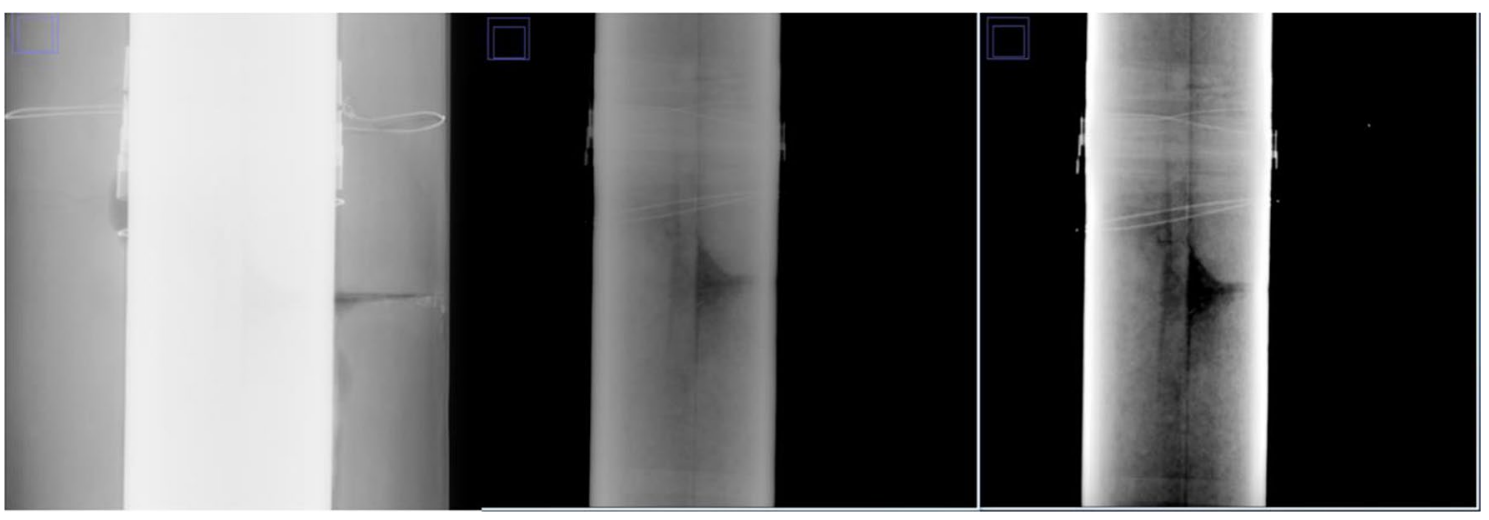

Fig. 3 Digital Radiographic filter utilization for optimized inspection

With the additional benefits of digital data, the ability for Assisted Analysis software tools may enhance the technician to focus on areas of interest and dispositioning versus mundane review of mostly acceptable images. The future may align with the medical radiographic process of autodetection in the examination phase. Establishing validation frameworks may increase adoption and confidence as the technology continues to advance. A few advantages may be in increased reliability, minimizing fatigue and potential oversight.

Although disruptive to the current ecosystem for both the onsite service provider and the asset owner, the convergence to the overarching migration support more expeditious transition for optimal operational benefits and increase data fidelity. The transitioned pressure of evaluating partners (inspection providers, equipment manufacturers, software and connected pathways) must be transparent for the collective benefits to operation and not a low-bid scenario to dominate.

\subsection{Use-Case: Expansion/New Construction Projects}

An estimated \$27.7 billion is active in CAPEX spend pipeline projects are at planning and engineering phase [24]. Businesswire, reported in March 2020 that $70 \%$ of the domestically produced petroleum products are expected to be transported by pipeline and there are currently 2.4 million miles of natural gas and 190,000 miles of liquid petroleum pipelines through the US [32]. Industry is actively engaging in efforts to embrace technological innovations that harness cost-reduction, increased regulatory compliance and advanced innovations. These activities present prospects to capitalize on the digital transformation and migration through status-quo of legacy inspection solutions into NDE 4.0 .

Opportunities exist to focus on a sub-segment in the new construction projects. Focusing on a high impact area of small diameter pipe weld quality inspection that still entrenched in film and force analog to digital migration obtained through a stepwise iteration that unlock a wide variety of productivity and performance benefits. The disruption of COVID-19 and projects being delayed/deferred, an evaluation through current business strategies may benefit by aligning throughout the value-chain. Specifically, the value-chain entailing asset owner; Engineering, Procurement and Construction (EPC), inspection company and equipment supplier to collaborate for ideal condition and can capitalize on the digital transformation through review of current written procedures on project lets and potentially re-negotiated contracts with the future in mind. Although potentially an initially higher per/weld rate to accommodate the hardware transition, the benefits on digital data, geospatial linkage, data storage, global collaboration and timeline compressions in overall project may justify the transition.

When seeking to understand the volume of radiographs, it is imperative to weigh the full value-proposition and not just per image impact. Price-point correlations may not consider the imbedded costs of chemical storage/disposal, processing time, dark-room requirements and storage when required on various projects that has been amortized over time. The lull of conventional norms almost nullifies the honest embedded inefficiencies and potential new capabilities like remote review and storage in bytes versus vaults. Industry must take a hard look at the full impacts of film processing, some considerations (not exhaustive) may help in consideration.

The start-up of a digital fleet may offer advantages over retrofitting conventional film business models. Outfitting a truck with darkroom, chemical bays and other required accessories may run upwards of $\sim \$ 150 \mathrm{~K} /$ unit for either film or CR, but the portability of DR may be managed with a panel, source and a laptop. This also allows for project portability from location centric rental. Additionally, in the fleet of X-ray trucks, some regions may have $50-150$ rigs containing multiple chemical reservoirs that must be stocked with processing chemicals; maintained for proper concentrations and contamination mitigations that in turn must manage the 
disposal costs that can easily be $\$ 6000 /$ month. Forgoing the processing time for film, DR offers fast onsite review within seconds and offering higher resolution enhanced through post processing enhancement tools.

Digital allows for real-time review minimizing re-shoots. It is stated that there is an average of $\sim 5 \%$ re-shoots on a given project, the impact may be burdensome requiring additional set-up and return to location yielding costly project delays. Digital also allows for global collaboration of digital image evaluations which can help in resource management flexibility and with the overall project oversight, scheduling and communication. In a tablet format, digital workflows can be created for or by the service provider's needs and support minimization of Human Factors and ensure repeatable performance. Additionally, the potential to utilization of Assisted Analysis tools support the maximum utilization of technician efforts and time. The overall project benefits with faster response to production and multiple locations globally, where film is a single hard image.

Two other important considerations are the storage of the images and potential benefit of reduction in exclusion zone. Film storage time requirement vary up to the life of the plant and are subject to fading over time and massive storage requirements either onsite or off-site for a fee with compounding charges. Accessibility imposes another burden if needed. With the sensitivity of the panels, there is the potential to reduce exclusion zone with lower doses and more image acquisitions while still yielding more sensitive images.

Disruption is not a task for the faint of heart, but clinging to a very profound quote from Wayne Dyer "If you change the way you look at things, the things you look at change" can drive our view of progress, adoption of NDE 4.0 may become a closer reality. In conclusion, we discuss how new digital technologies can revolutionize a wide range of routine operational and maintenance tasks for the oil and gas industry sectors. Every day, more and more technological breakthrough options will become available on the market and eventually may augment the human element for collaborative efficiencies elevating baseline. This digital dependence on computers, automation, and the many forms of artificial intelligence invoke transparency and training to increase operational efficiencies and profits globally throughout the industry.

\section{Tactical and Practical Aspects of Change Management: Human Factor}

NDE 4.0 has already demonstrated that there is a systematic integration of multiple technologies that had a direct affect upon how to plan and conduct radiography in all industries.
But there is another side to this progression that needs to be addressed and it deals with the "human element."

As the lines blur between film and digital radiography there is a critical need to develop and implement a plan of action that will address, the challenges of technology and NDE 4.0. Besides the technical component of the digital evolution we need to accept that one of the hidden catalysts for meaningful change is dependent upon its acceptance by organizations and the individuals that these changes will impact.

It's universally acknowledged that change is hard. Enterprise level change of digital transformation requires extensive changes within technology, training and certification, systems, processes, financial investments, and often company culture. Change is required of employees, partners, and sometimes customers as well [33]. The entire landscape of radiography testing is changing and one of these essential components of change starts with the NDE technician.

A recent survey [34] conducted by PQNDT states "Our most recent salary and benefits survey of the NDT industry was full of interesting facts. But one that stands out as worrisome was the average age of the respondents: 42 years, with more than 13 years of experience in the industry. Why worry about this figure? It shows a definite 'maturing' of the NDT workforce, which is a good thing in terms of experience and professionalism. But as NDT workers continue to age, is there sufficient young talent coming up behind them to fill a growing need?"

This survey points out an important question related to "is there sufficient young talent coming up behind them to fill a growing need?" [35]. This survey points out that not only is there a need for "sufficient young talent" to enter the NDE field in the upcoming years, but also the curriculum used to develop their skills must reflect the technological capability of the NDE Methods. Although beyond the scope of this paper the question related to the applicable NDE curriculum is applicable as it relates to our colleges and universities for the development of our workforce's future in design, research and engineering.

The evolution of Radiography Testing provides us the opportunity to see, experience and learn how multiple technologies and market demands have come together, highlighting the need for a holistic strategy that puts the human element at the core of meaningful changes. The Radiography Testing migration into the digital world demands that people involved in all levels of radiography testing refined their knowledge and skills to capitalize on the potential offered by technological integration.

New techniques such as computed tomography and resonant acoustic method are being used for what is called "full body inspections" provides an overall evaluation of a component are being used by NDE Testing Labs. Testing systems are becoming faster and cheaper as demand increases. The 
acceptance of these technologies and NDE applications will only accelerate in the upcoming years.

NDE 4.0 highlights the growing challenge that industry documents like, ASNT, SNT-TC-1A, ISO 9712 and others must adapt to the changing technology landscape for the qualification and certification of NDE personnel. These advancements demand a refreshed perspective of the needs for the NDE practitioners to learn and utilize digital radiography. What should or should not be included in a RT training program is beyond the scope of this paper however NDE 4.0 does open the door for constructive conversations of how industries can find a uniformed yet adaptable qualification/certification model that could be established as the foundation of moving forward.

As new flexible RT automated systems are integrated into the workplace, organizations must develop multiple strategies to develop the technician's ability to perform both the repeatable and different tasks. The NDE 4.0 RT technician will need to be trained to detect the complexities and subtleties that new technology has introduced into the inspection process.

A critical component of the RT qualification and certification process is the NDE trainer. The NDE trainers is on the front lines of the NDE 4.0 process and is challenged to find innovated ways to reach the technicians both in the classrooms and back on-the-job. There are signs that companies are moving in the right direction of creating processes to provide technical training and development programs related to digital radiography, but these changes are taking place in an isolated manner.

RT trainers need to master a range of soft skills that are normally not address in these sort of technical papers however for our efforts to be successful the RT trainers must have not only understand the new technologies but also command the skills to transfer these technologies into practical and measurable actions.

Besides their technical skills the trainers should also have the following three characteristics:

- Empathy individuals who teach advanced RT systems will need to display an understanding of the transitional issues related to traditional RT practices and the RT migration to advance technology-based applications.

- Adaptable individuals who teach advanced RT systems with the understanding that with the advent of more powerful technology tools, computers and data analysis capa- bilities we have entered an age of continuous updating of applications, tools, code and technician's skills.

- Integrated/Interaction individuals who help develop the technician's behavior on-the-job by using a combination of monitoring applications, coaching, and continuous training strategies.

During this 4.0 evolution there are no signs that digital radiography will do away with the framework that has been developed through years of film radiography. In other words, there will still be level I, II and IIIs and there will still be the need for interpreters needed for the transformation of NDE 4.0 into reality. There will continue to be the need for radiation safety, technical RT skill training, on-the-job experience and the need for a structured qualification and certification process.

Digital radiography is not tearing down the walls of film radiography testing. In fact, digital radiography is allowing us to build on a proven successful film radiography, a foundation that 4.0 can build upon.

Figure 4 illustrates that change is more than embracing a new technology. Change is personal and as digital radiography testing moves forward; we are reminded that many change efforts fail because people are insufficiently prepared for change. When people are not prepared, they are more likely to resist the change effort and less likely to function effectively under the new system or process.

From the company and organizational perspective there is a need to recognize that history shows that innovation could and has yielded greater inequality, particularly in its potential to disrupt the workforce. As automation substitutes for traditional inspection processes there will be a degree of displacement of workers by new hardware, software and inspection applications.

Organizations and companies have started the process of planning and investment into the strategy that will develop the workforce needed for today and tomorrows innovations in Radiography Testing. Radiography Testing is no exception. Figure 5 illustrates the cycle of relationships that are needed to fully realize and leverage NDE 4.0. These relationships along with technological innovations have challenged the way that we conduct radiography at every stage from design and engineering of products, managing the manufacturing process, planning inspection cycles, implementation of the testing process, evaluation of product compliance to

Fig. 4 Resistance and acceptance to change

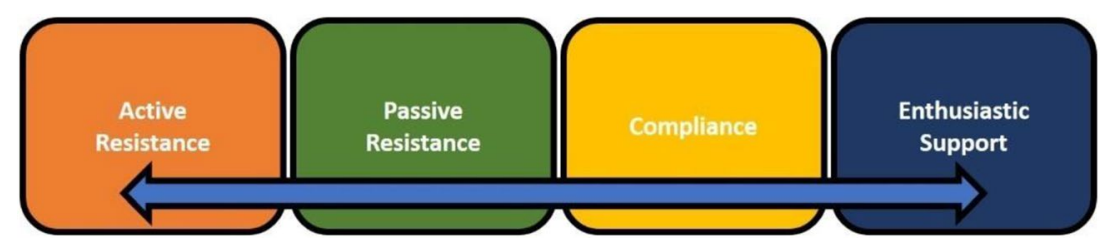




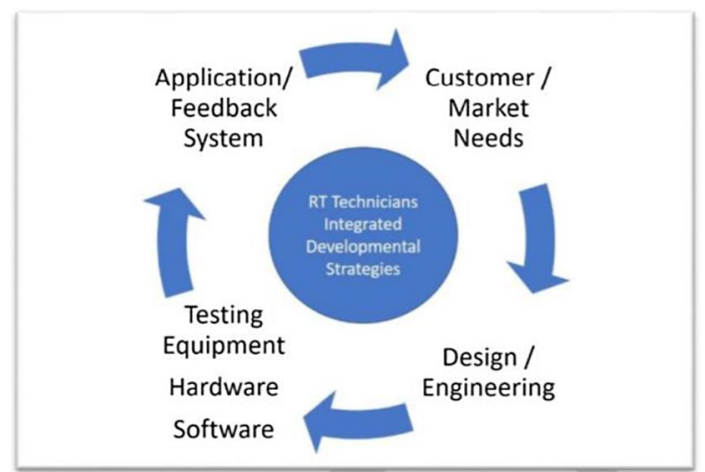

Fig. 5 Strategic cycle of technological integration

established codes and standards and the recording of the testing results.

What makes this even more of a challenge is that this evolution is not static, it is a dynamic process that will continue to occur. In other words, we need to remember that after NDE 4.0 will come 5.0 and so-on. As we explored earlier in this paper anyone using computed radiography and digital radiography will need an ongoing understanding of the new generation of equipment and software that makes up the Radiography Testing process. But that is only the start of what is needed to be taught to the 4.0 RT technicians and managers. We also are faced with developing systems and processes to deliver knowledge and skills to the appropriate people and in a timely manner.

When it comes to the radiography testing technicians and operators besides the traditional body of knowledge that are part of the accepted RT training outlines there needs to be a degree of knowledge about wireless and cloud technologies, networking, digital telecommunications networks, software applications, imaging screens technology, data transfer, GPS, 3D utilization, NDE monitoring techniques, and voice command controls.

These innovations are necessary in order for individuals to develop and adapt their skills to deal with the advancement of hardware, software and automation that will allow inspection systems to analyze millions of data points and use automatic defect recognition software to not only locate defects on a part, but also recognize what kind of defect.

Companies that acquire and cultivate new digital-related skills will have a distinct advantage as NDT 4.0 reshapes the industry, including identifying new roles for RT technicians to maximize their skills in data acquisition and analysis.

Figure 6 points out that when it comes to the Radiography Testing technicians and operators besides the traditional body of knowledge that are part of the accepted RT training outlines there needs to be a degree of knowledge about wireless and cloud technologies, networking, digital telecommunications networks, software applications, imaging screens

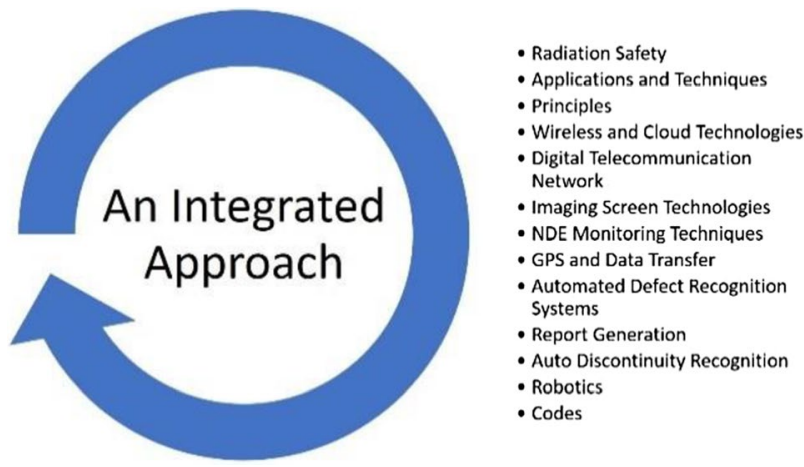

Fig. 6 Integrated skills development for NDE 4.0 radiography testing

technology, data transfer, GPS, 3D utilization, NDE monitoring techniques, and voice command controls.

In his paper titled "The Future of Non-Destructive Testing in the Context of Industry 4.0," Professor Randolf Hanke states, "In the future it won't be just a case of deciding whether a part is good or bad. Rather, it will be about providing customers with a monitoring system that shows them how they can optimize their processes" [36]. His statement provides the stark reality that we cannot separate Industry 4.0 from NDE 4.0.

Radiography Testing and similar nondestructive testing technologies along with personnel training and certification models will be on the front lines of making these predictions a reality in the NDE 4.0 environment. NDE 4.0 and the development of the technicians and managers will have a positive influence on the NDE field and will continue to grow as more companies are developing strategies to utilize these advances and challenges in the most efficient way possible.

\section{Conclusion}

In this manuscript we have explored how new digital technologies can revolutionize a wide range of routine operational and maintenance tasks for the oil and gas industry sectors is discussed. Every day, more and more technological breakthrough options will become available on the market and eventually may augment the human element. This digital technological dependence on computers, automation and the many forms of artificial intelligence is the new today and is being used to improve operations and increase company profits globally.

NDE 4.0 like Industry 4.0 has risen from interacting innovations. The rapid nature of changes in technology must be matched by the pace to develop and effectively adapt codes, standards and regulations needed to leverage the advancements in nondestructive testing. The potential gains from 
Table 1 General key considerations for Radiography options in the US oil and gas industry

\begin{tabular}{lll}
\hline & Film & CR \\
\hline Price per image & $\$ 0.85 /$ sheet of $4.5^{\prime \prime} \times 10^{\prime \prime}$ film & $\$ 0.1-\$ 1.5^{\mathrm{a}}$ \\
Equipment & NA for existing to $\sim \$ 150 \mathrm{~K} /$ unit for new setup & $\sim \$ 100 \mathrm{~K} /$ unit for equipment \\
Chemical Disposal cost & $\$ 6 \mathrm{k} /$ month & $\mathrm{NA}$ \\
Reshot rate & $\sim 5 \%$ & $\sim 0 \%$ (replaced by filtering and software enhancing tools) \\
Radiation exposure safety & Yes & Yes \\
Chemical exposure safety & Yes & $\mathrm{NA}$ \\
IT, computer trainings & NA & Yes \\
Response time & $\sim 30$ min for film processing (Automatic System) & Instant \\
Availability of RT technicians & Larger pool of qualified/experienced personnel & Smaller pool of qualified/experienced personnel \\
Average Hourly pay rate of & $35-75 \$ / \mathrm{h}$ & $50-90 \$ / \mathrm{h}$ \\
trained personnel & & \\
Training and certification & As per code specification and company procedures & As per code specification and company procedures \\
\hline
\end{tabular}

${ }^{a}$ Computed Radiographic (CR) plates can range in the $\$ 150$ per similar size plate; price per shot ranges from $\$ 0.10$ to $\$ 0.08$ cents/exposure for 1500-2000 exposures to with an average use of 100 exposure at $\$ 1.50$ per exposure

the migration to NDE 4.0 is so significant that makes this migration inevitable. Table 1 explores some of the key considerations of the Oil and Gas industry when deciding on the transition to NDE 4.0 in terms of Radiography.

Author contributions Not applicable.

Funding Not applicable.

Data Availability Not applicable.Code Availability Not applicable.

\section{Compliance with Ethical Standards}

Conflict of interest All the authors of this paper are working in the NDE industry.

\section{References}

1. Meyendorf, N., Heilmann, P., Bond, L.: NDE 4.0 in manufacturing: challenges and opportunities for NDE in the 21st century. Mater. Eval. 78, 794-803 (2020). https://doi.org/10.32548/2020. me-04144

2. Vrana, J.: NDE Perception and emerging reality: NDE 4.0 value extraction. Mater. Eval. 78, 835-851 (2020). https://doi. org/10.32548/2020.me-04131

3. Schulenburg, L.: NDT 4.0-opportunity or threat? (2019). https:// www.linkedin.com/pulse/ndt-40-opportunity-threat-lennart-schul enburg/

4. Singh, R.: Purpose and pursuit of NDE 4.0. Mater. Eval. 78, 784793 (2020). https://doi.org/10.32548/2020.me-04143

5. John, A.: Intelligence augmentation and human-machine interface best practices for NDT 4.0 reliability. Mater. Eval. 78, 869-879 (2020). https://doi.org/10.32548/2020.me-04133

6. Schulenburg, L.: NDT 4.0: opportunity or threat? Mater. Eval. 78, 852-860 (2020). https://doi.org/10.32548/2020.me-04134
7. Ramezani, M.G., Hasanian, M., Golchinfar, B., Saboonchi, H.: Automatic boiler tube leak detection with deep bidirectional LSTM neural networks of acoustic emission signals. In: Zonta, D., Huang, H. (eds.) Sensors and Smart Structures Technologies for Civil, Mechanical, and Aerospace Systems 2020, p. 56. SPIE, Bellingham (2020)

8. Pascu, L.: Roaming agreements help NB-IoT adoption in Europe, North America (2020). https://www.edgeir.com/roaming-agree ments-help-nb-iot-adoption-in-europe-north-america-20200601

9. Kirchof, N.: Killed off by NTT DoCoMo: NB-IoT still kicking globally, but for how long? (2020). https://www.iot-now. com/2020/05/11/102743-killed-off-by-ntt-docomo-nb-iot-still -kicking-globally-but-for-how-long/

10. ISO 12052:2017: Health informatics_-digital imaging and communication in medicine (DICOM) including workflow and data management. In: Book of Standards Volume: 35.240 Applications of Information Technology. ISO (2017)

11. ASTM E2339-15: Standard Practice for Digital Imaging and Communication in Nondestructive Evaluation (DICONDE). In: Book of Standards Volume: 03.03. ASTM International, West Conshohocken (2015)

12. Grigorescu, S., Trasnea, B., Cocias, T., Macesanu, G.: A survey of deep learning techniques for autonomous driving. J. Field Robot. 37, 362-386 (2020). https://doi.org/10.1002/rob.21918

13. Baomar, H., Bentley, P.J.: An Intelligent Autopilot System that learns flight emergency procedures by imitating human pilots. In: 2016 IEEE Symposium Series on Computational Intelligence, SSCI 2016 (2017). https://doi.org/https://doi.org/10.1109/ SSCI.2016.7849881

14. Higaki, T., Nakamura, Y., Tatsugami, F., Nakaura, T., Awai, K.: Improvement of image quality at CT and MRI using deep learning. Jpn. J. Radiol. 37, 73-80 (2019). https://doi.org/10.1007/ s11604-018-0796-2

15. Luo, W., Phung, D., Tran, T., Gupta, S., Rana, S., Karmakar, C., Shilton, A., Yearwood, J., Dimitrova, N., Ho, T.B., Venkatesh, S., Berk, M.: Guidelines for developing and reporting machine learning predictive models in biomedical research: a multidisciplinary view. J. Med. Internet Res. 18, e323 (2016). https://doi. org/10.2196/jmir.5870

16. Litjens, G., Kooi, T., Bejnordi, B.E., Setio, A.A.A., Ciompi, F., Ghafoorian, M., van der Laak, J.A.W.M., van Ginneken, B., Sánchez, C.I.: A survey on deep learning in medical image 
analysis. Med. Image Anal. (2017). https://doi.org/10.1016/j. media.2017.07.005

17. Montagnon, E., Cerny, M., Cadrin-Chênevert, A., Hamilton, V., Derennes, T., Ilinca, A., Vandenbroucke-Menu, F., Turcotte, S., Kadoury, S., Tang, A.: Deep learning workflow in radiology: a primer. Insights Imaging 11, 22 (2020). https://doi.org/10.1186/ s13244-019-0832-5

18. Chartrand, G., Cheng, P.M., Vorontsov, E., Drozdzal, M., Turcotte, S., Pal, C.J., Kadoury, S., Tang, A.: Deep learning: a primer for radiologists. RadioGraphics 37, 2113-2131 (2017). https://doi. org/10.1148/rg.2017170077

19. Ghosh Mondal, T., Jahanshahi, M.R., Wu, R., Wu, Z.Y.: Deep learning-based multi-class damage detection for autonomous postdisaster reconnaissance. Struct. Control Health Monit. (2020). https://doi.org/10.1002/stc.2507

20. Akbar, M.A., Qidwai, U., Jahanshahi, M.R.: An evaluation of image-based structural health monitoring using integrated unmanned aerial vehicle platform. Struct. Control Health Monit. 26, e2276 (2019). https://doi.org/10.1002/stc.2276

21. Adhikari, R.S., Bagchi, A., Moselhi, O.: Automated condition assessment of concrete bridges with digital imaging. Smart Struct. Syst. 13, 901-925 (2014). https://doi.org/10.12989/ sss.2014.13.6.901

22. Ebrahimkhanlou, A., Dubuc, B., Salamone, S.: A generalizable deep learning framework for localizing and characterizing acoustic emission sources in riveted metallic panels. Mech. Syst. Signal Process. 130, 248-272 (2019). https://doi.org/10.1016/j.ymssp .2019 .04 .050

23. Jahanshahi, M.R., Chen, F.-C.: Methods and systems for Crack Detection (2020). http://patft.uspto.gov/netacgi/nph-Parse $\mathrm{r}$ ? Sect $1=\mathrm{PTO} 2 \&$ Sect $2=$ HITOFF $\& \mathrm{p}=1 \& \mathrm{u}=\% 2$ Fnet $\mathrm{ahtml}$ $\% 2$ FPTO $\% 2$ Fsearch-bool.html\& $\mathrm{r}=1 \& \mathrm{f}=\mathrm{G} \& \mathrm{l}=50 \& \mathrm{co} 1=\mathrm{AND}$ $\& d=$ PTXT\&s $1=$ Jahanshahi\&s2 $=15,606,921 \& O S=$ Jahanshahi $+\mathrm{AND}+15,606,921 \& \mathrm{RS}=$ Jahanshahi+AND+15,606,921

24. ResearchAndMarkets.com: Global IoT in Oil and Gas Market (2019 to 2024)-Focus on Solutions, Applications and Industry Stream (2020). https://www.businesswire.com/news/home/20200 623005661/en/Global-IoT-Oil-Gas-Market-2019-2024
25. Editor, O. \& G.I.: What does IIoT mean for oil and gas? (2019). https://www.oilandgasiq.com/integrity-hse-maintenance/news/ what-is-the-iiot

26. Simon Cushing, R.M.: Why IT Operating Models are Under Strain and How Oil and Gas CIOs Should Respond (2018)

27. Associated Press Archive: Check this Out: Deposit Money by Taking a Photo (2010). https://www.technologyrevie w.com/2010/02/23/122514/check-this-out-deposit-money-bytaking-a-photo/

28. Lecun, Y., Bottou, L., Bengio, Y., Ha, P.: Gradient based learning applied to document recognition. Proc. IEEE 48(11), 1-46 (1998)

29. Schulenburg, L.: Introduction to Digital Radiography systems and its operating principles (2017). https://www.linkedin.com/pulse/ introduction-digital-radiography-systems-its-lennart-schulenburg/

30. Schulenburg, L.: Digital Radiography in Aerospace (2018). https ://www.linkedin.com/pulse/digital-radiography-aerospace-lenna rt-schulenburg/

31. NACE International: NACE Study Estimates Global Cost of Corrosion at \$2.5 Trillion Annually. NACE International (2016). https ://inspectioneering.com/news/2016-03-08/5202/nace-study-estim ates-global-cost-of-corrosion-at-25-trillion-ann

32. ResearchAndMarkets.com: Global Oil and Gas Pipeline Fabrication and Construction Market-Growth, Trends, and Forecast (2020 to 2025) (2020). https://www.businesswire.com/news/ home/20200318005420/en/Global-Oil-Gas-Pipeline-Fabricatio n-Construction-Market

33. Atkinson, R.D., Stephen, E.: The Manufacturing Evolution: How AI Will Transform Manufacturing and the Workforce of the Future (2019)

34. Where Will Our Future Techs Come From? (2019). https://www. pqndt.com/where-will-our-future-techs-come-from/

35. Path, Y., Job, P., Here, S., Path, Y., Job, P., Here, S.: Salary Survey (2019)

36. Hanke, R.: The Future of Nondestructive Testing in the Context of Industry 4.0. (2018). https://www.ndt.net/search/docs. php3?id $=22507$ 